\title{
First dedicated observations of runaway electrons in the COMPASS tokamak
}

\author{
Miloš Vlainić, \\ Jan Mlynáŕ, \\ Vladímir Weinzettl, \\ Richard Papřok, \\ Martin Imríšek, \\ Ondřej Ficker, \\ Petr Vondráček, \\ Josef Havlíček
}

\begin{abstract}
Runaway electrons present an important part of the present efforts in nuclear fusion research with respect to the potential damage of the in-vessel components. The COMPASS tokamak a suitable tool for the studies of runaway electrons, due to its relatively low vacuum safety constraints, high experimental flexibility and the possibility of reaching the H-mode D-shaped plasmas. In this work, results from the first experimental COMPASS campaign dedicated to runaway electrons are presented and discussed in preliminary way. In particular, the first observation of synchrotron radiation and rather interesting raw magnetic data are shown.
\end{abstract}

Key words: plasma diagnostics • runaway electrons • tokamak

M. Vlainić ${ }^{凶}$

Department of Applied Physics,

Ghent University,

Sint-Pietersnieuwstraat 41, B9000 Gent, Belgium,

Tel.: +420774536639,

E-mail: vlaki77@gmail.com or milos.vlainic@ugent.be

\section{J. Mlynár, O. Ficker}

Institute of Plasma Physics ASCR

Za Slovankou 3, 18200 Prague 8, Czech Republic

and Faculty of Nuclear Sciences and Physical Engineering,

Czech Technical University in Prague,

7 Břehová Str., 11519 Prague, Czech Republic

V. Weinzettl, P. Vondráček

Institute of Plasma Physics ASCR,

Za Slovankou 3, 18200 Prague 8, Czech Republic

R. Papřok, M. Imríšek, J. Havlíček

Institute of Plasma Physics ASCR,

Za Slovankou 3, 18200 Prague 8, Czech Republic

and Faculty of Mathematics and Physics,

Charles University in Prague,

Prague, Czech Republic

Received: 12 June 2014

Accepted: 20 October 2014

\section{Introduction}

Electrons in a tokamak are accelerated by toroidal electric field, while Coulomb interaction with a charged plasma particles results in a frictional force. For high electron velocities the frictional force decreases, with approximately quadratic dependence on the velocity. Thus for the given accelerating field there is some value of velocity (called the critical velocity) for which the accelerating force and the frictional force become equal. Electrons which achieve velocities higher than the critical velocity start to be accelerated continuously join the population of the so-called runaway electrons (RE).

RE can be generated in two main ways: 1) the primary (Dreicer) mechanism is mostly encountered in low density cold plasmas in the presence of the toroidal electric field; 2) the secondary (avalanche) mechanism comes into play when primary RE are transferring their energy via Coulomb collisions to the background (thermal) electrons.

In small/medium tokamaks (e.g. COMPASS, TCV) the REs are created shortly after the plasma breakdown due to high electric field or during current flat-top in the low density discharges (reasons for this behavior are explained further on in this article). On the contrary, in large/reactor-like tokamaks (e.g. in JET, and very probably in ITER) the REs arise mainly during disruptions ${ }^{1)}$ in connection

\footnotetext{
1) Sudden (and undesired) termination of confined plasma is called a disruption. It starts with a shorter phase called thermal quench (loss of the thermal energy of plasma), followed by a longer phase called current quench (loss of the magnetic energy of plasma).
} 
to the current quench phase. The REs can drag significant amount of pre-disruptive plasma current and achieve enormous energy that can severely damage the plasma facing components (PFC) and the first wall $(\mathrm{FW})$ as a result of a local RE deposition. This is the main reason why the runaways have to be suppressed in ITER and in future commercial nuclear fusion reactors [1].

Generally, tokamak operators try to anticipate the RE production in order to avoid possible damages of the vessel. Discharge scenarios with significant population of runaways, necessary for detailed studies of the RE, can severely damage in-vessel components of a tokamak, which can ultimately lead to a permanent vacuum or water leak and a prolonged shut down of the facility. This is a good motivation for efforts leading to better understanding of the process, but on the other hand, this is a big limitation for the detailed experimental studies. However, the COMPASS machine has relatively low plasma current in comparison to larger devices. Therefore, the RE cannot achieve very high energies and cause a severe damage to the vessel, which makes COMPASS a suitable machine for this kind of studies.

The COMPASS tokamak is a small size experimental device, with the major radius $R=0.56 \mathrm{~cm}$ and the minor radius $a=0.23 \mathrm{~cm}$. The toroidal magnetic field varies in the range of $0.9-2.1 \mathrm{~T}$, and the plasma current can reach up to $400 \mathrm{kA}$. The plasma configuration varies from circular and elliptical to $\mathrm{D}$-shaped plasmas, with a typical pulse length of $0.4 \mathrm{~s}$. Densities are flexible and are typically of the order of magnitude of $10^{19}$ electron $/ \mathrm{m}^{3}$ [2].

The article is structured as follows. Firstly, a brief introduction to the RE physics is presented in the next section. Then, section 'Diagnostics for RE' is devoted to diagnostic techniques used in the COMPASS tokamak for RE observation. The first dedicated observations of RE in COMPASS tokamak are reported in section 'First observations'. Finally, the last section contains few concluding sentences about the reported results and a general comment on the necessary diagnostics required for detailed statistical studies of RE.

\section{The RE physics}

Historically, REs were first observed by Wilson (the inventor of the cloud chamber) in his investigations of thunderclouds and thunderstorms [3]. RE have an important role in explaining astrophysical, atmospheric and solid state physics phenomena [4]. REs are not strictly a fusion plasma phenomenon but rather a more general physical phenomenon that can appear in variety of plasmas. Regarding the tokamaks, which are currently the most promising devices for future fusion reactor of all the magnetic fusion devices, REs were observed since the very beginning of their research.

As it was already mentioned in the introduction, REs are created when the accelerating force (produced by the electric field $\vec{E}$ ) is larger than the

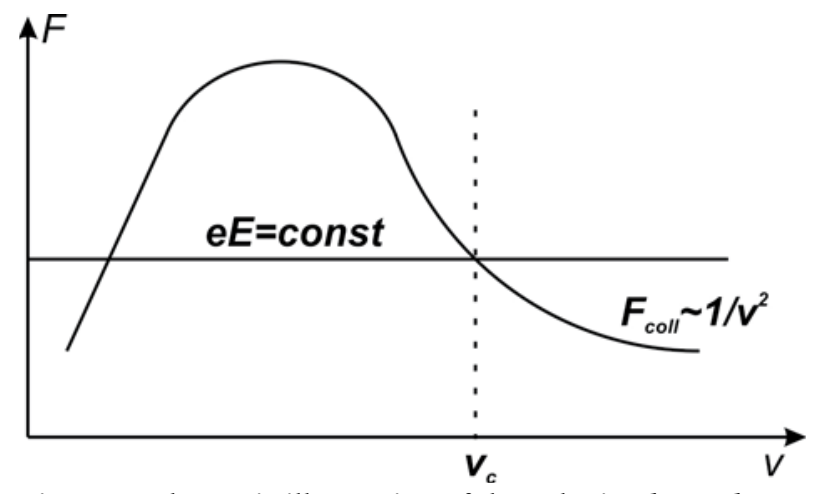

Fig. 1. A schematic illustration of the velocity dependence of the main accelerating $(e \vec{E})$ and frictional $\left(F_{\text {coll }}\right)$ forces in a tokamak. The critical velocity $v_{c}$ is marked with a dashed vertical line.

frictional force (produced by Coulomb collisions with charged background particles), which is schematically presented in Fig. 1. In tokamaks electrons accelerated in this way can reach energies of the order of magnitude of tens or hundreds of $\mathrm{MeV}$. Such highly energetic electrons can cause severe damage to the PFC and even penetrate into the FW material and melt it.

Dreicer electric field, derived in [5], $E_{\mathrm{D}}=$ $\left\{\left(n_{e} e^{3} \ln \Lambda\right) /\left(4 \pi \varepsilon_{0}^{2} T_{e}\right)\right\}$, represents a measure of the electric field required when electron velocity increases and exceeds the most probable random speed in plasma along one mean free path. This does not mean that RE cannot be created for smaller values of the electric field, since the Joule heating ${ }^{2)}$ itself can randomly lead to a high velocity of the electrons; it is only less probable for RE to occur and their population number will be significantly smaller. This is the reason why there are REs in real tokamaks even if the strength of the electric field never reaches the Dreicer value. A non-relativistic analysis was taken for the calculation of the Dreicer field, but if relativistic effects are taken into account then a different expression for the critical (threshold) electric field $E_{C}=\left\{\left(n_{e} e^{3} \ln \Lambda\right) /\left(4 \pi \varepsilon_{0}^{2} c^{2}\right)\right\}$ is obtained [6]. The critical electric field $E_{C}$ represents the lower limit for the RE generation.

Dreicer field is one of the parameters showing up in expression for the above mentioned critical velocity: $\left(v_{C} / v_{T e}\right)=\left(2+Z_{\text {eff }}\right)^{1 / 2}\left(E_{\mathrm{D}} / E_{\text {par }}\right)^{1 / 2}$, which is here normalized to the thermal velocity $v_{T e}$ of the background electrons, and $E_{p a r}$ is the electrical field parallel to the magnetic field. The equation is derived for a $1 \mathrm{D}$ case, in the direction of the magnetic field. It is obvious that this picture is highly idealized and it was shown in [7] that it is suitable for the electric fields far from the Dreicer field. In [7] a 2D model was developed and stochastic effects of Coulomb collisions were taken into account. This leads to a slight modification of the $v_{C}$ estimate: $\left(v_{C} / v_{T e}\right)=$ $\left(2+Z_{\text {eff }}\right)^{1 / 4}\left(E_{\mathrm{D}} / E_{p a r}\right)^{1 / 2}$. Finally, after introducing the critical velocity, the critical (kinetic) energy can be represented as: $W_{C} \mu\left(2+Z_{e f f}\right)^{1 / 2}\left(n_{e} / E_{p a r}\right)$. Now it can be seen that by keeping low electric field or high density, the critical energy is increased and RE

\footnotetext{
2) As it is a statistical process.
} 
generation is suppressed. Also the impurity factor $Z_{\text {eff }}$ plays some role, but due to the square root it has a weaker impact on the RE generation.

Generation of RE described in the two previous paragraphs corresponds to the so-called primary (Dreicer) mechanism. When a primary generated RE hits a background electron, the RE can transfer a significant amount of energy to this electron, so that it is also transferred to the 'runaway' region in the velocity space. REs generated in this way are called secondary (avalanche) REs. The RE avalanche process is actually a real danger for the future fusion reactors (e.g. ITER, DEMO), as their number increases exponentially with time. For example, the exponential gain due to the avalanche effect is expected to be $10^{13}$ in ITER [8].

Regarding the RE mitigations strategies, the best option would be if some kind of a real-time control of the RE beam ${ }^{3)}$ with magnetic field would be feasible. At present this is beyond our current knowledge and technical capability, but fusion community has been developing the necessary tools. The next step that could be done is to suppress the multiplication factor from the avalanche effect. This can be done directly or indirectly. A direct procedure could be based on the RE de-confinement, again with the help of magnetic fields. This has been done in some devices (e.g. DIII-D, FTU), but efficiency is far from $100 \%$. Anyway, this option can ultimately lead to the full control of REs. An indirect avalanche suppression is based on a massive gas injection (MGI), which leads to an increase of the critical electric field $E_{C}$. For the moment this method gives best results, but it leads to a plasma disruption as well.

It should be mentioned that REs are not necessarily harmful. A special kind of a discharge, the so-called slide-away, is an experimentally observed regime where REs are beneficial for plasma confinement [9]. In this regime RE are supporting the creation of a supra-thermal electron population which drives most of the plasma current, and loop voltage drops, while the plasma confinement is improved [10].

\section{Diagnostics for RE}

As already mentioned, REs were observed already in the first toroidal plasma experiments and their physical basis was studied in detail for typical tokamak discharges, as discussed in the previous section. Nevertheless REs still represent a significant challenge for disruptions created in future fusion reactors. Nowadays, to increase the understanding of the underlying relevant processes, there are numerous diagnostic options available or under development, as well as modeling research. Modeling is outside of the framework of this article, but interested reader is encouraged to read Refs. [7, 8]. Even so, the experimental study of REs is a challenging task as all those diagnostics have limited performances and the data analysis has to be done carefully.

\footnotetext{
3) $\mathrm{RE}$ beam is a term for a compact beam composed of significant amount of RE.
}

Generally, REs can be detected by various methods, see e.g. [11]:

- Soft X-ray (SXR) emission from the plasma and from the targets.

- Hard X-ray (HXR) emission from the plasma and from a thick target inside or outside the plasma.

- High excitation threshold line (like K or L lines from heavy impurities).

- Electromagnetic emission around $\omega_{c e}$ (typically in the $100-\mathrm{GHz}$ range) from low energy REs.

- Electromagnetic emission around $\omega_{\mathrm{LH}}$ (typically in the $\mathrm{GHz}$ range).

- Neutrons from the nuclear photoreactions in the plasma and particularly in targets outside the plasma.

- Thomson scattered laser light (which is Doppler-shifted as a result of the fast circulating runaways).

- Synchrotron radiation generated by relativistic particles.

- Cherenkov radiation generated by the escaped REs.

- Magnetic diagnostics for the RE energy, current and position estimations.

In this section both the commonly used indirect and direct diagnostic techniques for RE observation would be presented, together with the corresponding physical background. Namely, the tools for the measurement of SXR and HXR, photoneutrons, Cherenkov detector and synchrotron radiation are discussed, while the magnetic diagnostics will be only briefly mentioned. These diagnostics were used at the COMPASS tokamak for the RE observation.

\section{X-ray radiation}

When REs escape from the confined plasma, they hit $\mathrm{PFC} / \mathrm{FW}$ and as a consequence of this interaction emit the HXR bremsstrahlung. Thus a high intensity of the HXR signal is a sign of high runaway losses. The HXR diagnostic technique can be indirectly used for estimation of the RE energy distribution [12], if the detector has an energetic calibration and works as pulse height analyzer (PHA). To achieve PHA the detector must be well colimated, which is also beneficial for studies of spatial distribution of the escaped REs. The HXR radiation originates in many processes in the tokamak device. Hence, it is not easy to interpret the corresponding signals.

An SXR measurement is also applicable in order to follow the RE-relevant processes, and it can be used for measuring escaped and in-flight REs. The post-disruption runaways can interact with the residual plasma (bremsstrahlung) and the heavy metal impurities (radiation from their K- or L-shell). This technique is used in JET to follow the time evolution of the RE location during a disruption. For example, in [13] in Fig. 6 the time evolution of the height of the runaway beam can be clearly seen. Interaction of RE with PFC or diagnostic probes can be studied with 2D SXR cameras. The SXR signal interpretation encounters the same problems as HXR due 
to its various radiation origins in the tokamak. Moreover, SXR is strongly shielded by the metallic vacuum vessel.

\section{Particle detection}

A photoneutron is a neutron emitted from a nucleus excited by a high energy photon. Energetic photons can be produced as bremsstrahlung due to collisions of highly energetic REs interacting with the PFC/FW. However, the signal interpretation suffers the same problems as with HXR and SXR signals, as there are also other possible sources of neutron radiation in tokamaks and neutrons are easily scattered.

$\mathrm{RE}$ can easily reach relativistic velocities which exceed the speed of light in certain materials. Therefore, propagation REs in those materials results in the emission of the Cherenkov radiation. If such a material (diamond, aluminum nitride, etc.) is used inside light-protected measuring head at the edge plasma, the intensity of the Cherenkov radiation related to RE population can be measured. A simple setup of Cherenkov detectors used for example in the COMPASS, TORE SUPRA and ISTTOK tokamaks provided time evolution of the integral Cherenkov radiation emitted by electrons with a wide range of energies (above several hundred $\mathrm{keV}$ ), giving the information primarily about the presence of REs, rather than their number and energy, which makes it more a qualitative than a quantitative tool. When the detector is equipped with more channels with entrance windows of different thickness, the energy distribution can be directly measured [14]

\section{Synchrotron radiation}

Another possibility for detection of the REs is to monitor their synchrotron radiation. The synchrotron radiation is produced by relativistic charged particles accelerated in the presence of the magnetic field. The synchrotron radiation generated by REs in a tokamak has a continuous spectrum and usually achieves peak intensity in the infrared region. As the synchrotron radiation is not isotropic, being emitted preferentially in the direction parallel to the RE velocity - the so-called headlight effect - it should be observed with a tangential view. A 2D camera in the IR or in the visible spectrum can be used for measurements of the position, pitch angle and vertical velocity, while an IR spectrometer is more suitable for the energy, RE intensity, and transfer rate estimates $[15,16]$.

Synchrotron radiation is a promising diagnostic technique for the RE studies. However, since the detected intensity strongly depends on the particle energy, pitch angle, point of generation (plasma parameters), as well as spatial and energy distribution of runaways, a comparison of the measured and the calculated signals is quite difficult. In this respect it should be noted that spatial and energy distributions have never been experimentally determined; for diagnostic purposes a Gaussian or a uniform spatial distribution of the runaway beam is usually assumed and the energy distribution is often taken to be monotonic or uniform. Regarding the energy distribution, in Ref. [17] it is demonstrated that a realistic energy distribution can have important effects on the shape of the synchrotron spectrum. It was shown that simplification of the problem may result in an overestimation of the particle energy, which leads to completely wrong estimations of the RE number and transformation rate. Furthermore, highly collimated radiation in the direction of movement is characteristic for relativistic particles. Thus, diagnostics has to be positioned in (or close to) the $\mathrm{RE}$ plane for proper measuring.

\section{Magnetic diagnostics}

Magnetic diagnostics is a fundamental diagnostic method in the fusion devices. It is used for a real-time control of plasma position and shape, as well as for various other measurements (e.g. plasma current, loop voltage, plasma energy, etc.) while it is typically not used for the RE observation. Details on numerous magnetic diagnostics in COMPASS can be found in [18].

\section{First observations}

The first campaign dedicated to REs was carried out at the COMPASS tokamak and in this section some of the first observations are presented. The main characteristics of plasma discharges during this campaign was that plasmas were limited, circular and elliptical. Plasma current and plasma density were assumed varying values, but in general were relatively low. Scan was done across various parameters, such as plasma density, plasma position, gas puff and plasma current. Some of the experiments were realized with opposite (reversed) plasma current to check RE diagnostics, as some of diagnostics setup depend on the direction of the RE flow. The experimental setup of the diagnostics used in this campaign is schematically presented in Fig. 2 .

The observation of the time evolution of spectrally resolved HXR is one of the routine diagnostics used at COMPASS. Diagnostics consists of $\mathrm{NaI}(\mathrm{Tl})$ scintillator combined with a photomultiplier. In the recent campaign dedicated to the RE studies three additional $\mathrm{NaI}(\mathrm{Tl})$ scintillators were set around the torus at the distance of approximately $5 \mathrm{~m}$ from the tokamak and $2.5 \mathrm{~m}$ above the ground. Their toroidal distribution was approximately 90 degrees in between neighboring ones. Measurements recorded by four HXR detectors are shown in Fig. 3. A clear periodic behavior of the observed instabilities can be seen in this figure. Each of the instabilities seems not to be simultaneous around the torus, but there is some time delay of the peaks in the direction of the electron flow. On the basis of this delay it is possible to deduce the velocity of the event and possibly correlate it with some other known phenomenon in plasma. Furthermore, the reason why such event 


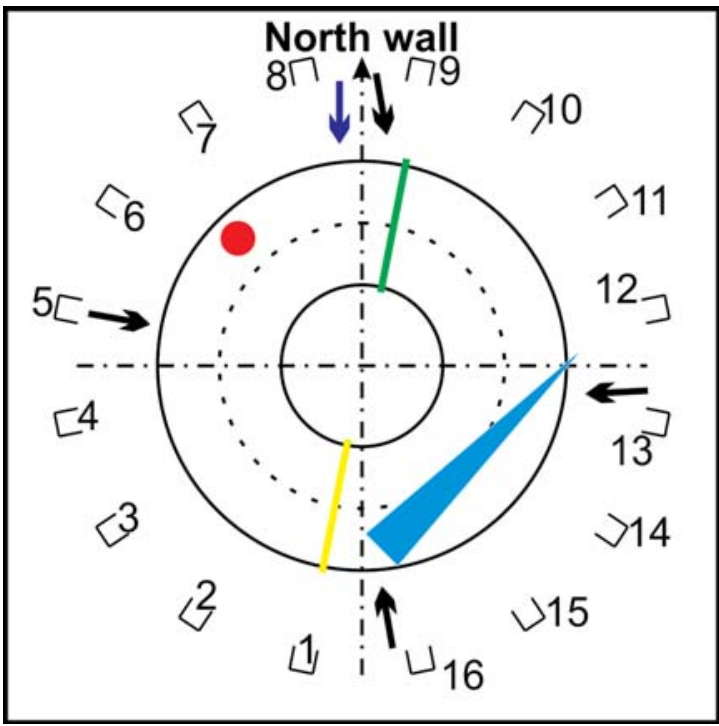

Fig. 2. An experimental setup of diagnostics used for the RE observations. The HXR detector orientations are marked as black arrows, while the photoneutron detector is marked as a dark blue arrow. The light blue triangle represents the approximate line of sight of the IR camera. The red circle is a port with the Cherenkov detector. The yellow line is the Rogowski coil used for plasma current measurements, while the green line denotes the location of the IPRs.

disappears after making one toroidal motion is yet to be evaluated, as it could also have been caused by a technical delay. Different behavior of the signals is due to setup and detector differences. Anyway, physical interpretation of this observation is still to be evaluated. This is the reason why in the future new equivalent detectors will be purchased.

At COMPASS, detection of fast neutrons is done with zinc sulfide phosphor scintillator embedded in a plastic matrix. Unfortunately, neutron detectors based on scintillators are sensitive to strong HXR radiation, which makes the interpretation of this signal a challenging task. Improvement is under consideration and main options are diamond or bubble detectors.

A single-channel Cherenkov detector (red circle in Fig. 2) mounted on a fixed manipulator was also used for RE measurements at COMPASS, see Fig. 5 in

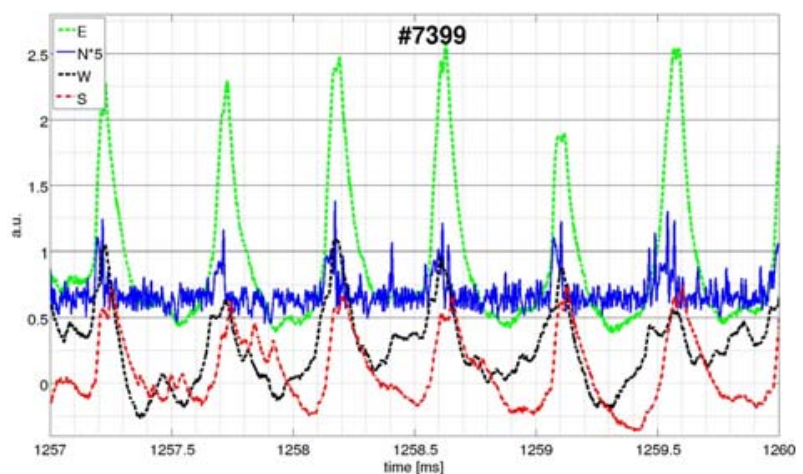

Fig. 3. HXR signals recorded in the discharge \#7399. The signal from detectors located near the east wall is indicated in green, and the signal from the detector at the north wall is indicated in blue. Close to the signal from the west wall there is a black signal and a red signal coming from the detector located next to the south wall.
[19]. This technique can be used for measurements of radial and poloidal distribution of REs [20,21]. The multi-channel Cherenkov detector is currently under development at the IPPLM (Poland) for the upcoming $\mathrm{RE}$ campaigns at COMPASS.

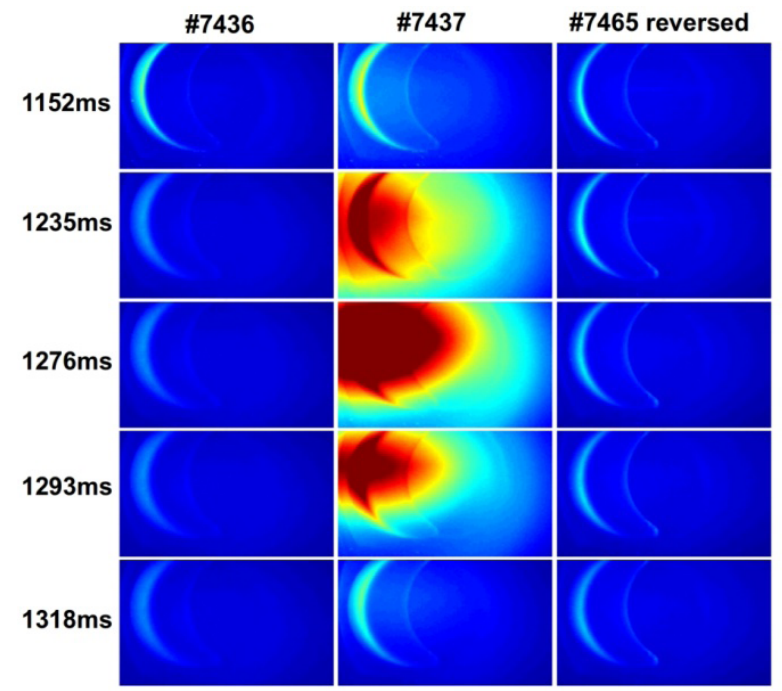

Fig. 4. A tangential view recorded by the IR camera for the three different discharges at five different times. Measurements correspond to the time when an intensive HXR signal was emitted, and the last one corresponds to the beginning of the current ramp-down phase. The discharge \#7436 (left) is a discharge for which no synchrotron radiation was observed, while the discharge \#7437 (middle) was accompanied by synchrotron radiation. The discharge \#7465 (right) was performed with reversed plasma current, or in other words when electrons were moving away from the IR camera.
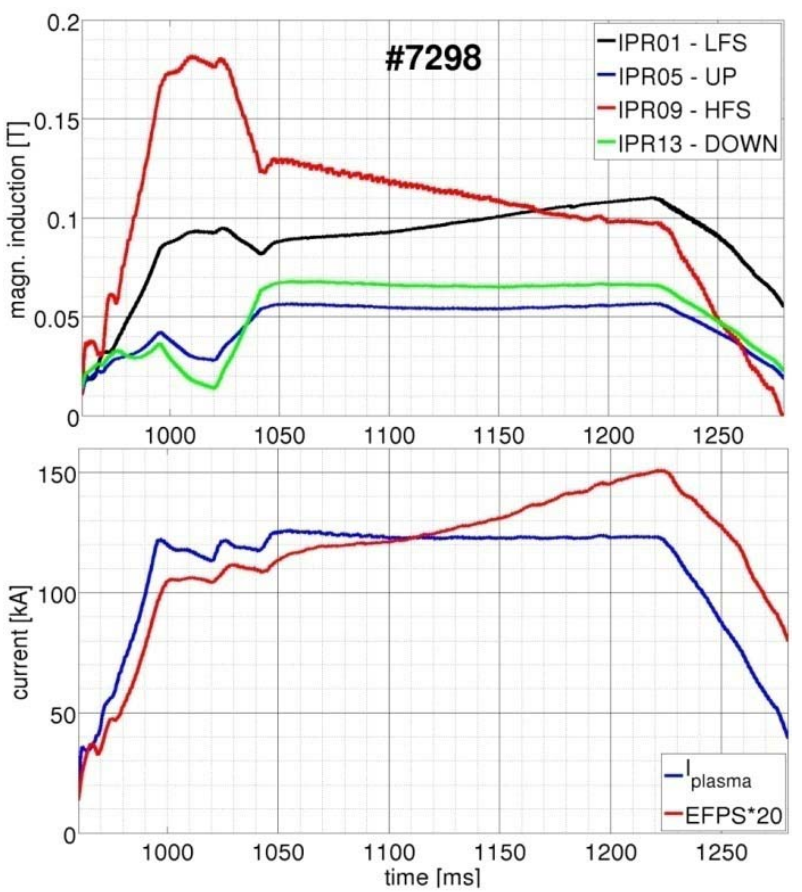

Fig. 5. Raw magnetic data collected by: (upper part) LFS IPR (black), top IPR (blue), HFS IPR (red) and bottom IPR (green); (lower part) the plasma current measured by the Rogowski coil and the EFPS vertical control field. Note: EFPS was recalibrated, so that it can be compared with the plasma current. 
The IR camera (light blue triangle in Fig. 2) used for the already mentioned experiments is sensitive to the radiation in the range of $7.5-13 \mu \mathrm{m}$. Its speed is $100 \mathrm{~Hz}$, with a bolometer integration time of $10 \mathrm{~ms}$. An uncoated $\mathrm{ZnSe}$ window has been used for the experiments with a transmittance of around 70\% at the given range. Camera was set in the tangential view and the diameter of the observed region in the plasma core region was approximately $15 \mathrm{~cm}$. The IR camera measured the synchrotron radiation from RE, although only a small region of plasma core was observed with it. In Fig. 4 the results from three different discharges and for five different times during the discharge are presented. The left one is an example of a discharge with no signal, because plasma density was relatively high and the RE did not have enough energy to radiate in the IR range covered by the camera. The middle one is an example of the observed synchrotron radiation, where time of observation corresponds to the expulsion of intensive HXR. It is important to point out that the camera did not saturate, but the scale was changed for better insight. The right column gives an example with low density but reversed plasma current, where no signal is observed due to the fact that electrons are moving away from the camera.

In our measurements raw signals were collected from the internal partial Rogowski coils (IPR - green in Fig. 2), the equilibrium field power supply (EFPS) and the plasma current (yellow in Fig. 2). The COMPASS tokamak is equipped with 16 IPRs distributed in the poloidal direction around the torus. EFPS is the major vertical control field that pushes plasma towards high field side (HFS) for stability reasons. Plasma current is measured with the Rogowski coil. As the RE are driving an important part of the plasma current, they affect magnetic diagnostics.

Interesting magnetic observations regarding REs are illustrated in Fig. 5. In the beginning, a constant plasma current during flat-top phase should be observed at the lower part of the figure. It is obvious from the upper part of the figure that the signal of IPR at the low field side (LFS) is increasing, while the signal from IPR at HFS is decreasing during the current flat-top. The other two IPRs are located at the bottom and the top of the vessel and they are kept constant during the current flat-top phase. A possible conclusion is that there is some structure moving outwards. As a result the EFPS is increasing, which leads to pushing of the plasma inwards and trying to keep structure confined and stable. This 'structure' could be RE beam that is driving significant part of the measured plasma current. This observation requires further discussion and evaluation. What is sure that this behavior is in conflict with the EFIT ${ }^{4)}$ reconstruction.

4) EFIT is a calculation that iteratively solves the Grad-Shafranov equation and gives information about magnetic configuration, plasma shape, current and pressure profile, etc.

\section{Conclusions}

During the first RE campaign at the COMPASS tokamak interesting features were observed during the experiments. For the moment, we have only observations which are waiting for further investigation, discussion and evaluation.

Some of these observations are shown in this article. At the moment we can say that some toroidal asymmetry is probably observed with HXR signals. Furthermore, REs in COMPASS seem to have higher velocity than previously expected, as synchrotron radiation was observed with IR at rather low wavelength range for previously reported energies.

In general, systematic (but extremely careful) studies of the RE discharges are possible nowadays, even though Finken predicted differently in Ref. $[15]^{5}$. Namely for high RE energy the synchrotron radiation has the largest potential as the RE study tool. Otherwise, SXR (array or 2D camera), multi-channel Cherenkov detector and spatially distributed HXR detectors give better insight into physics and behavior of REs, into their generation, confinement and loss. Beside diagnostics, for the full insight in the REs behavior a proper modeling, theory and data analysis are all necessary. One of the current main goals of the tokamak community is to achieve complete avoidance of the RE generation. If RE must occur anyway, then ITER should be ready for their mitigation or control. Finally if all this failed, at least expectations on the deposition heat loads, and damage localization should be known so that the maintenance is more efficient.

Acknowledgments. This work has been carried out within the framework of the EUROfusion Consortium and has received funding from the Euratom research and training programme 2014-2018 under grant agreement no. 633053 . The views and opinions expressed herein do not necessarily reflect those of the European Commission. Also to the project MSMT LM2011021 from which a COMPASS operation is supported. Hereby, I would like to give special thanks for the COMPASS team that made those experiments real. A lot of thanks to Faculty of Nuclear Sciences and Physical Engineering (Czech Technical University) for lending us the HXR detectors and making the measurements possible. We appreciate help around Cherenkov detector done by Polish colleagues from the National Centre for Nuclear Research (Otwock-Swierk, Poland).

\section{References}

1. Kikuchi, M., Lackner, K., \& Tran, M. Q. (2012). Fusion physics (pp. 352-353). Vienna: IAEA.

2. Pánek, R., Bilyková, P., Fuchs, V., Hron, M., Chráska, P., Pavlo, P., Stockel, J., Urban, J., Weinzettl, V., Zajac, J., \& Zacek, F. (2006). Reinstallation of

\footnotetext{
${ }^{5)}$ In an article that introduced synchrotron radiation as a possible diagnostic technique for the RE it is claimed that "systematic studies of runaway discharges are generally not possible".
} 
the COMPASS-D tokamak in IPP ASCR. Czech. J. Phys., 56(Suppl. 2), B125-B137. DOI: 10.1007/ s10582-006-0188-1.

3. Wilson, C. T. R. (1925). The acceleration of $\beta$-particles in strong electric fields such as those of Thunderclouds. Proc. Cambridge Philos. Soc., 22(04), 534-538. DOI: 10.1017/S0305004100003236.

4. Solis, J. R. (2012, November 20). Disruption and runaway electrons in tokamaks. Lecture notes distributed in the unit Plasma Physics and Fusion Seminars, Universidad Carlos III de Madrid, Madrid, Spain.

5. Dreicer, H. (1959). Electron and ion runaway in a fully ionized gas I. Phys. Rev., 115(2), 238-249. DOI: 10.1103/PhysRev.115.238.

6. Connor, J. W., \& Hestie, R. J. (1975). Relativistic limitations on runaway electrons. Nucl. Fusion, 15(3), 415-424. DOI: 10.1088/0029-5515/15/3/007.

7. Fernandez-Gomez, I., Martin-Solis, J. R., \& Sanchez, R. (2012). Perpendicular dynamics of runaway electrons in tokamak plasmas. Phys. Plasmas, 19, 102504. DOI: 10.1063/1.4757644.

8. Eriksson, L. -G., Helander, P., Andersson, F., Anderson, D., \& Lisak, M. (2004). Current dynamics during disruptions in large tokamaks. Phys. Rev. Lett., 92(20), 205004. DOI: 10.1103/PhysRevLett.92.205004.

9. Lu, H. W., Hu, L. Q., Li, Y. D., Zhong, G. Q., Lin, S. Y., Xu, P., \& EAST Team. (2010). Investigation of fast pitch angle scattering of runaway electrons in the EAST tokamak. Chinese Phys. Lett., 19(12), 125201. DOI: 10.1088/1674-1056/19/12/125201.

10. Chen, Z. Y., Wan, B. N., Ling, B. L., Gao, X., Du, Q., Ti, A., Lin, S. Y., \& Sajjad, S. (2007). Runaway electron beam instability in slide-away discharges in the HT-7 tokamak. Chinese Phys. Lett., 24(11), 3195-3198. DOI: 10.1088/0256-307X/24/11/048.

11. Knoepfel, H., \& Spong, D. A. (1979). Runaway electrons in toroidal discharges. Nucl. Fusion, 19(6), 785-829. DOI: 10.1088/0029-5515/19/6/008.

12. Papřok, R., Havlíček, J., Hron, M., Janky, F., Krlín, L., Stökel, J., \& Kocmanová, L. (2012). Runaway electrons in COMPASS tokamak. In WDS'12 Proceedings, 29 May - 1 June 2012 (pp. 228-232). Prague, Czech Republic: Charles University.

13. Gill, R. D., Alper, B., de Baar, M., Hender, T. C., Johnson, M. F., Riccardo, V., \& contributors to the EFDA-
-JET Work programme. (2000). Behavior of disruption generated runaways in JET. Nucl. Fusion, 40(8), 1039-1044. DOI: 10.1088/0029-5515/42/8/312.

14. Jakubowski, L., Plyusnin, V. V., Sadowski, M. J., Zebrowski, J., Malinowski, K., Rabiński, M., Fernandes, H., Silva, C., Duarte, P., \& Jakubowski, M. (2012). Estimation of ISTTOK runaway-electrons energies by means of a Cherenkov-type probe with modified AIN radiators. Nukleonika, $57(2), 177-181$.

15. Finken, K. H., Watkins, J. G., Rusbüldt, D., Corbett, W. J., Dippel, K. H., Goebel, D. M., \& Moyer, R. A (1990). Observation of infrared synchrotron radiation from tokamak runaway electrons in TEXTOR. Nucl. Fusion, 30(5), 859-870. DOI: 10.1088/0029$5515 / 30 / 5 / 005$.

16. Jaspers, R. (1995). Relativistic runaway electrons in tokamak plasmas. Doctoral thesis, Eindhoven University, The Netherlands.

17. Stahl, A., Landreman, M., Papp, G., Hollmann, E. \& Fulop, T. (2013). Synchrotron radiation from a runaway electron distribution in tokamaks. Phys. Plasmas, 20, 093302. DOI: 10.1063/1.4821823.

18. Havlíček, J., \& Hronová, O. (2010). Magnetic diagnostics of COMPASS tokamak. Retrieved June 5th 2014, from http://www.ipp.cas.cz/Tokamak/euratom/index.php/en/compass-diagnostics/magneticdiagnostics.

19. Papřrok, R., Krlín, L., \& Stökel, J. (2013). Observation and prediction of runaway electrons in the COMPASS tokamak. In WDS'13 Proceedings, 4-7 June 2013 (pp. 60-66). Prague, Czech Republic: Charles University.

20. Jakubowski, L., Sadowski, M. J., Stanislawski, J., Malinowski, K., Zebrowski, J., Jakuwoski, M., Weinzettl, V., Stökel, J., Vacha, M., \& Peterka, M. (2007). Application of Cherenkov detectors for fast electron measurements in CASTOR-tokamak. In 34th EPS Conference on Plasma Physics, 2-6 July 2007 (P5.097). Warsaw, Poland: European Physical Society.

21. Jakubowski, L., Sadowski, M. J., Stanislawski, J., Malinowski, K., Zebrowski, J., Jakuwoski, M., Weinzettl, V., Stökel, J., Vacha, M., \& Peterka, M. (2008). Cherenkov detector for measurements of fast electrons in CASTOR-tokamak. In AIP'08 Conference Proceedings, 22-24 October 2007 (pp. 219-223). Lisbon, Portugal: American Institute of Physics. 